\title{
Should resection extent be decided by total lesion size or solid component size in ground glass opacity-containing lung adenocarcinomas?
}

\author{
Boyu Lin", Rui Wang", Liang Chen, Zhitao Gu, Chunyu Ji, Wentao Fang \\ Department of Thoracic Surgery, Shanghai Chest Hospital, Shanghai Jiao Tong University, Shanghai, China \\ Contributions: (I) Conception and design: B Lin, R Wang, W Fang; (II) Administrative support: W Fang; (III) Provision of study materials or patients: \\ R Wang, Z Gu, C Ji; (IV) Collection and assembly of data: B Lin, L Chen; (V) Data analysis and interpretation: All authors; (VI) Manuscript writing: \\ All authors; (VII) Final approval of manuscript: All authors. \\ \#These authors contributed equally to this work. \\ Correspondence to: Wentao Fang. Department of Thoracic Surgery, Shanghai Chest Hospital, Shanghai Jiao Tong University, Shanghai 200030, \\ China. Email: vwtfang@hotmail.com.
}

Background: Indication for sublobar resections in early-stage lung adenocarcinomas has been
controversial. The purpose of this study was to find appropriate selection criteria for sublobar resections in
ground glass opacity (GGO)-containing early-stage lung adenocarcinomas.
Methods: We retrospectively studied 985 consecutive patients with clinical stage IA, peripheral
GGO-containing lung adenocarcinomas $\leq 3 \mathrm{~cm}$ in size. According to their radiological appearance, they were
divided into a pure GGO group and a part-solid nodule (PSN) group. The PSN group was further divided
into a GGO-predominant subgroup and a solid-predominant subgroup. Propensity-score matching (PSM)
was conducted first in PSNs with similar total lesion size and then in those with similar solid component
size to eliminate potential confounders. Histological characteristics and prognosis were compared between
matched patients to investigate the prognostic value of total lesion size and solid component size. Then
solid component size was chosen as the selection criterion to compare the prognosis of patients receiving
lobectomy or sublobar resections. Results: Comparing to PSNs, pure GGO lesions had significantly more favorable histological characteristics and prognosis, with 100\% 5-year overall survival (OS), even though $33.3 \%$ of patients with pure GGO lesions $>20 \mathrm{~mm}$ in total lesion size received sublobar resections. For 157 pairs of PSNs with similar total lesion size but different solid component size after the first PSM, the solid-predominant subgroup had significantly worse histological characteristics and prognosis than the GGO-predominant subgroup. After the second PSM, histological characteristics and prognosis were comparable between 73 pairs of PSNs with similar solid component size but different total lesion size. Multivariable analysis showed that solid component size, rather than total lesion size or consolidation-to-tumor ratio (CTR), was an independent prognostic factor. For PSNs containing solid component size $\leq 2 \mathrm{~cm}$, relapse-free survival (RFS) was similar after sublobar resections or lobectomy $(95.0 \%$ vs. $93.6 \%, \mathrm{P}=0.592)$. The results remained similar for PSNs of total lesion size $>2 \mathrm{~cm}$ but solid component size $\leq 2 \mathrm{~cm}(88.9 \%$ vs. $90.0 \%, \mathrm{P}=0.893)$.

Conclusions: Solid component size better predicts histological characteristics and prognosis than total lesion size in early-stage GGO-containing lung adenocarcinomas. Instead of total lesion size, solid component size $\leq 2 \mathrm{~cm}$ may be a more appropriate selection criterion for sublobar resections in such patients.

Keywords: Lung adenocarcinomas; ground glass opacity (GGO); sublobar resections

Submitted Feb 19, 2021. Accepted for publication Apr 25, 2021.

doi: $10.21037 /$ tlcr-21-132

View this article at: http://dx.doi.org/10.21037/tlcr-21-132 


\section{Introduction}

Lobectomy has been regarded as the standard procedure for surgical treatment of stage IA non-small cell lung cancer (NSCLC) since a randomized study comparing lobectomy and sublobar resections was published in 1995 (1). The Lung Cancer Study Group (LCSG) trial demonstrated that local recurrence rate was 3 -fold higher in the limited resection group compared to that in the lobectomy group. However, with the use of computed tomography (CT) scan for screening, an increased proportion of early-stage lung cancers, especially ground glass opacity (GGO)-containing nodules, are detected nowadays. And recent retrospective studies reported that sublobar resections might have similar efficacy as lobectomy on prognosis in such GGOcontaining early-stage NSCLC patients and might help preserve more pulmonary function (2-6). Therefore, the conclusion from the LCSG trial needs to be reexamined given the paradigm shift of surgical candidates. The current National Comprehensive Cancer Network (NCCN) guideline for NSCLC also suggests that in addition to being a palliative procedure for patients who cannot tolerate lobectomy, sublobar resections may also be considered as an intentional treatment in selected patients with a peripheral nodule $\leq 2 \mathrm{~cm}$ in size and one of the following criteria: pure adenocarcinoma in situ histology, radiologic surveillance confirming a long doubling time ( $\geq 400$ days), or $\geq 50 \%$ ground-glass appearance on CT scan (7).

Two prospective randomized trials, JCOG0802 and CALGB140503, comparing sublobar resections and lobectomy are still underway $(8,9)$. Both trials use total lesion size $\leq 2 \mathrm{~cm}$ as inclusion criterion. The JCOG0802 trial compares segmentectomy with lobectomy for tumors of total lesion size $\leq 2 \mathrm{~cm}$ and consolidation-to-tumor ratio $($ CTR) $>0.5$ (8), while the CALGB 140503 trial compares sublobar resections with lobectomy for tumors of total lesion size $\leq 2 \mathrm{~cm}$, without a definite inclusion criterion on tumor histology or their CT appearance (9). However, recent studies revealed that solid component size appeared to be a better predictor for malignant behaviors than total lesion size in lung adenocarcinomas $(10,11)$. In the eighth edition of the American Joint Commission on Cancer (AJCC) TNM staging system, clinical T1 category for partsolid nodule (PSN) has also been changed from using total lesion size previously to using solid component size (12). This means that PSNs with similar solid component size but different total lesion size or CTR are now categorized into the same $\mathrm{T}$ stage. Thus, it seems inappropriate to still use total lesion size or CTR to guide surgical procedure for GGO-containing nodules.

We thus aimed to study whether total lesion size or solid component size should be applied in the selection of sublobar resections for early-stage GGO-containing lung adenocarcinomas. We present the following article in accordance with the STROBE reporting checklist (available at http://dx.doi.org/10.21037/tlcr-21-132).

\section{Methods}

\section{Study population}

From a prospectively kept database, 2,093 consecutive patients who underwent surgery with a curative intention for clinical stage IA lung adenocarcinomas appearing as small $(\leq 3 \mathrm{~cm})$, peripheral, GGO-containing nodules at Shanghai Chest Hospital between January 2013 and July 2015 were retrieved for the study. The inclusion criterion was a pathologic diagnosis of lung adenocarcinomas appearing as small GGO-containing nodules $(\leq 3 \mathrm{~cm})$ on thin-slice CT scan. The exclusion criteria were patients having CT scan at other hospitals, an interval of over 1 month between CT examination and surgery, and nodules too irregular to measure precisely. A peripheral nodule was defined as the center of the nodule within the outer third of the lung field. According to the radiological appearance of the nodules on CT scan, patients were divided into a pure GGO group and a PSN group. The PSN group was further divided into a GGO-predominant subgroup and a solid-predominant subgroup according to their CTR less or greater than 0.5 . The study was conducted in accordance with the Declaration of Helsinki (as revised in 2013). This study was approved by the institutional review board of the Shanghai Chest Hospital [No. KS(Y)1668] and informed consent was waived due to the retrospective nature of the study.

\section{Radiological and pathological evaluations}

CT scans were performed from the apex to the base of the lung at suspended maximum inspiration using a 64-detector CT row scanner (Brilliance 64; Philips, Eindhoven, The Netherlands). Soft tissue and lung algorithms with a thickness of $1 \mathrm{~mm}$ were used to reconstruct the images. Consolidation was defined as a homogeneous increase in pulmonary parenchymal attenuation that obscures the margins of airway walls and vessels, while GGO was defined 
as hazy increased opacity of lung, with preservation of bronchial and vascular margins (13). All measurements were conducted with a lung window setting (lung window center, $-520 \mathrm{HU} / \mathrm{lung}$ window width, 1,450 HU). The longest diameters of the total lesion and the consolidation part were measured on the planes displaying the largest lesion and the largest portion of solid component, respectively. Histological types of the tumors were classified according to the International Association for the Study of Lung Cancer (IASLC) /American Thoracic Society (ATS)/ European Respiratory Society (ERS) classification of lung adenocarcinomas as adenocarcinoma in situ, minimally invasive adenocarcinoma and invasive adenocarcinoma (14).

\section{Surgery and postoperative follow-up}

All patients received video-assisted thoracoscopic surgery. Lobectomy, segmentectomy and wedge resection were selected according to the surgeons' decision with an intention-to-treat. Sublobar resections were considered when a parenchymal resection margins $\geq 2 \mathrm{~cm}$ or $\geq$ the size of the nodule could be obtained. Patients received either systematic mediastinal lymph node sampling or systematic lymph node dissection, according to the surgeons' decision. Systematic nodal sampling was carried out according to the ESTS guideline for intraoperative lymph node staging (15). And systematic dissection aimed to remove all mediastinal tissue together with the lymph nodes within anatomical landmarks (16). Patients were followed every 6 months for the first 2 years after surgery and then once a year from the third to the fifth year. Physical examination, chest CT scan, ultrasonography of abdominal, cervical and supraclavicular regions and serum tumor markers were checked routinely. Magnetic resonance imaging of brain, bone scans and PETCT were reserved only if recurrence or metastasis was suspected.

\section{Statistical analysis}

Statistical analyses were performed using the SPSS Statistics 23.0 software (SPSS Inc., Chicago, IL, USA) and R software (version 3.6.3, http://www.r-project.org). Continuous variables were compared with the Student $t$-test or Wilcoxon rank-sum test, depending on whether the variables were normally distributed. Categorical variables were analyzed with the Chi-square test or the Fisher exact test when appropriate. Relapse-free survival (RFS) was defined as the time between the day of surgery and the day of first recurrence or last follow-up. Overall survival (OS) was defined as the time between the day of surgery and the day of death or last follow-up. Deaths from other causes were considered as censored. RFS and OS were calculated with Kaplan-Meier curves and compared by log-rank test. In order to investigate the prognostic value of total lesion size and solid component size while reducing the effects of confounding biases, we compared the prognosis and histological characteristics between the GGO-predominant subgroup and the solid-predominant subgroup by means of two propensity-score matched analyses. We first compared patients with nodules of similar total lesion size but different solid component size, and then compared those of similar solid component size but different total lesion size. Other clinicopathological characteristics including sex, age, smoking history, American Society of Anesthesiologists (ASA) score, body mass index (BMI), forced expiratory volume in 1 second (FEV1) and resection extents were incorporated into propensity-score matching (PSM) to eliminate potential confounding factors. A ratio of 1:1 match with a caliper size of 0.04 was applied for PSM, using the nearest-neighbor matching algorithm without replacement. Potential risk factors for prognosis were analyzed by Cox proportional hazards regression model. All tests were two tailed with a statistical significance of 0.05 .

\section{Results}

According to the selection criteria, a total of 985 patients with lung adenocarcinomas manifesting as GGOcontaining nodules $\leq 3 \mathrm{~cm}$ in size on CT scan were enrolled in this study. Based on the radiological appearance, they were divided into a pure GGO group $(\mathrm{n}=613)$ and a PSN group ( $\mathrm{n}=372)$. The clinicopathological characteristics are shown in Table 1. There were significant differences in age, smoking history, total lesion size and resection extents between the two groups. Compared to the PSN group, the pure GGO group showed significantly lower grade histological subtypes $(\mathrm{P}<0.001)$, less lymphovascular invasion $(0 \%$ vs. $2.4 \%, \mathrm{P}<0.001)$, less visceral pleural invasion $(0.2 \%$ vs. $5.1 \%, \mathrm{P}<0.001)$ and less lymph node metastasis $(0 \%$ vs. $5.4 \%, \mathrm{P}<0.001)$. In the pure GGO group, only $45.4 \%$ and $4.7 \%$ of patients received lobectomy and systematic lymph node dissection, respectively. There were $33.3 \%$ of patients with pure GGO >20 mm in total lesion size who received sublobar resections. With a median follow-up of 55 months, the pure GGO group had significantly better 5-year RFS (99.8\%, 95\% CI: $99.4-$ 
Table 1 Clinicopathological characteristics of 985 patients with small GGO-containing lung adenocarcinomas

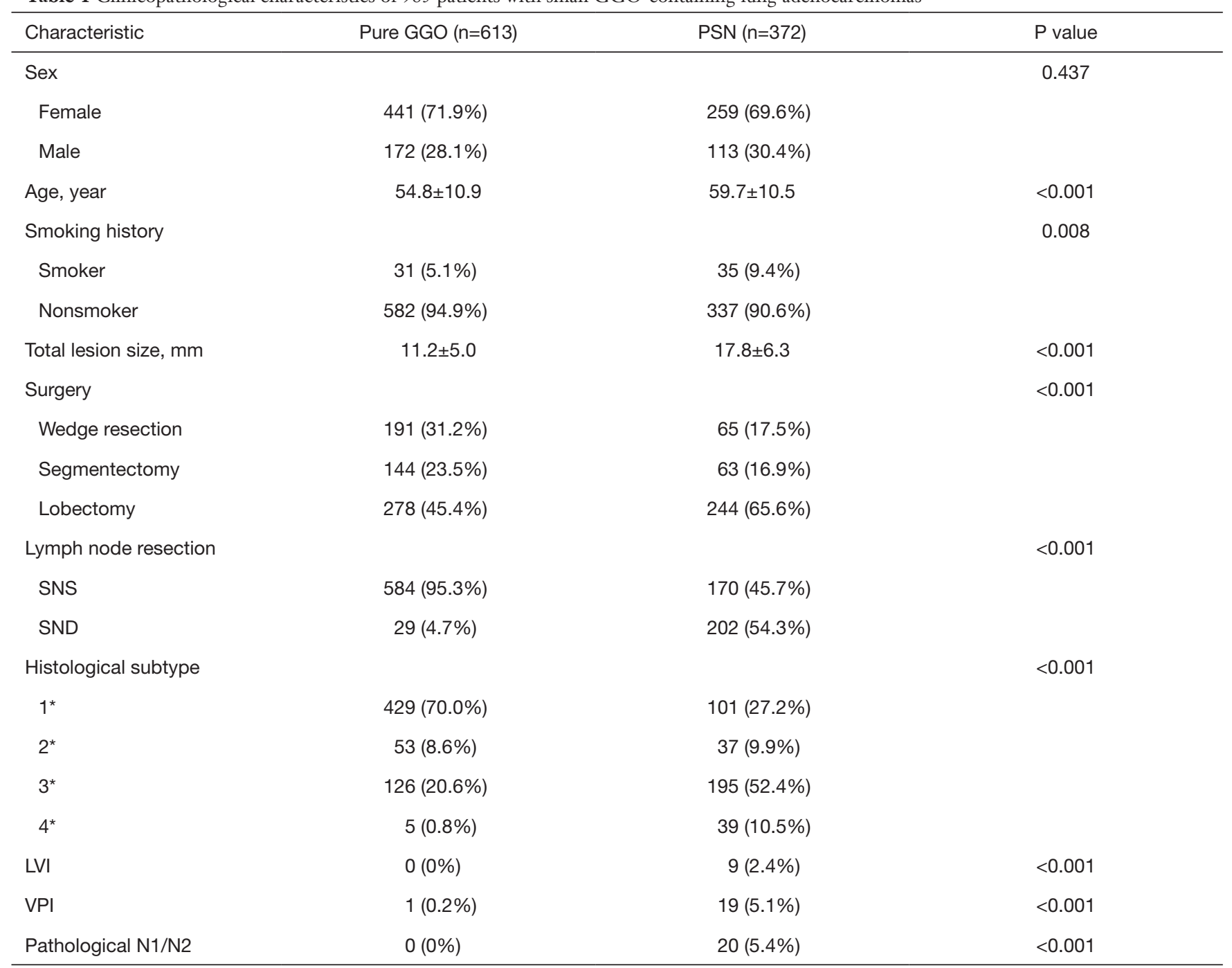

GGO, ground glass opacity; PSN, part-solid nodule; SNS, systematic lymph node sampling; SND, systematic lymph node dissection; $1^{*}$, adenocarcinoma in situ/Minimally invasive adenocarcinoma; $2^{\star}$, lepidic predominant, excluding micropapillary and solid component; $3^{\star}$, acinar/papillary predominant, excluding micropapillary and solid component; $4^{\star}$, micropapillary/solid component; LVI, lymphovascular invasion; VPI, visceral pleural invasion.

$100 \%$ vs. $93.0 \%, 95 \%$ CI: $90.5-95.5 \%, \mathrm{P}<0.001)$ and 5 -year OS (100\% vs. $98.3 \%$, 95\% CI: 96.9-99.7\%, $\mathrm{P}=0.001)$ than the PSN group (Figure 1).

In the PSN group, significant differences in clinicopathological characteristics including age, smoking history, ASA score, solid component size, total lesion size, and resection extents were observed between the GGOpredominant subgroup and the solid-predominant subgroup before PSM (Table 2). But no significant difference was observed except for solid component size between the two subgroups after the first PSM, and no significant difference was observed except for total lesion size after the second PSM (Table 3).

To analyse the impact of solid component size on outcomes, 157 pairs of PSNs with similar total lesion size were obtained after the first PSM (Table 3). The solidpredominant subgroup had a similar total lesion size $(18.6 \pm 6.3$ vs. $17.9 \pm 6.1 \mathrm{~mm}, \mathrm{P}=0.340)$ but a significantly larger solid component size than the GGO-predominant subgroup $(12.7 \pm 5.0$ vs. $6.5 \pm 2.9 \mathrm{~mm}, \mathrm{P}<0.001)$. And there were significantly more invasive adenocarcinomas in the solid-predominant subgroup than in the GGO-predominant 
A

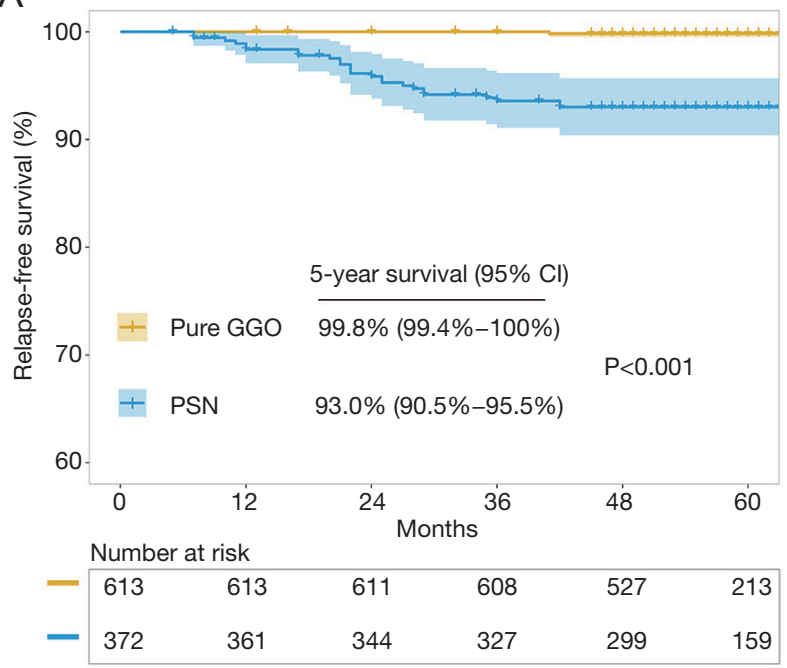

B

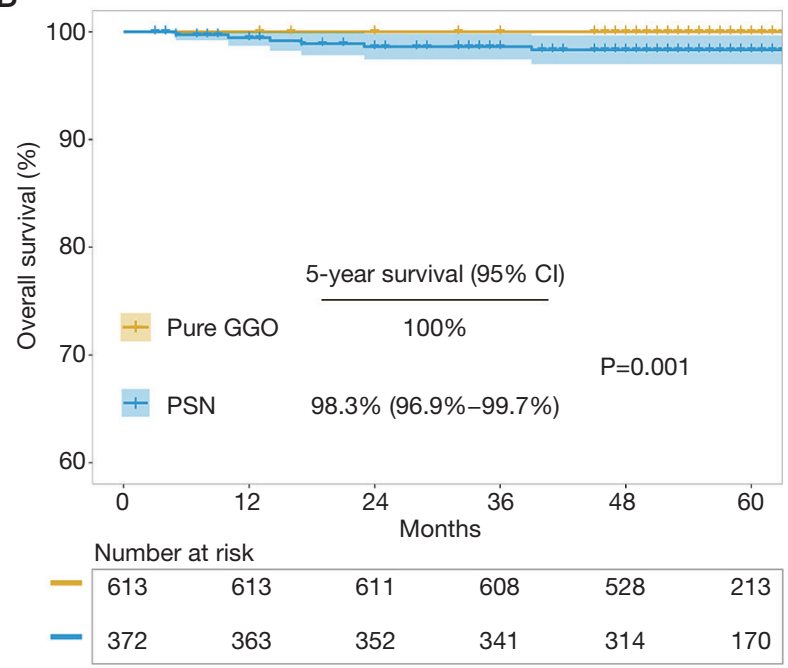

Figure 1 Comparison of survival between the pure ground glass opacity (GGO) group and part-solid nodule (PSN) group. (A) The pure GGO group had significantly better 5 -year relapse-free survival $(\mathrm{P}<0.001)$ than the PSN group; (B) the pure GGO group had significantly better 5-year overall survival $(\mathrm{P}=0.001)$ than the PSN group.

subgroup. Also, significantly more lymphovascular invasion, visceral pleural invasion and lymph node metastasis were observed in the solid-predominant subgroup (Table 3). Although the resection extents were similar, the solidpredominant subgroup had significantly worse 5 -year RFS (89.2\%, 95\% CI: $84.1-94.3 \%$ vs. $98.0 \%$, 95\% CI: $95.8-$ $100 \%, \mathrm{P}=0.001)$ and 5 -year OS $(96.7 \%, 95 \% \mathrm{CI}: 93.8-$ $99.6 \%$ vs. $100 \%, \mathrm{P}=0.023)$ than the GGO-predominant subgroup (Figure 2).

To analyse the impact of total lesion size on outcomes, 73 pairs of PSNs with similar solid component size were obtained after the second PSM (Table 3). The solidpredominant subgroup had a similar solid component size ( $8.8 \pm 2.8$ vs. $8.7 \pm 2.6 \mathrm{~mm}, \mathrm{P}=0.878$ ) but significantly smaller total lesion size $(14.0 \pm 4.6$ vs. $21.3 \pm 5.6 \mathrm{~mm}, \mathrm{P}<0.001)$ than the GGO-predominant subgroup. For these tumors with similar solid component size, the solid-predominant subgroup had similar histological characteristics (invasive adenocarcinomas, lymphovascular invasion, visceral pleural invasion and lymph node metastasis) to the GGOpredominant subgroup (Table 3). Besides, there was no significant difference in RFS (97.1\%, 95\% CI: $93.2-100 \%$ vs. $95.7 \%, 95 \%$ CI: $91.0-100 \%, \mathrm{P}=0.637)$ between the solid-predominant subgroup and the GGO-predominant subgroup (Figure 3) and 5-year OS was $100 \%$ in both subgroups receiving similar extent of resections.

Upon multivariate analysis in the PSN group, solid component size (for solid component size $>10$ and $\leq 20 \mathrm{~mm}$, $\mathrm{HR}=4.577,95 \%$ CI: $1.457-14.382, \mathrm{P}=0.009$; for solid component size $>20$ and $\leq 30 \mathrm{~mm}, \mathrm{HR}=6.351,95 \%$ CI: $1.515-26.628, \mathrm{P}=0.011)$, lymphovascular invasion $(\mathrm{HR}=4.266,95 \%$ CI: $1.367-13.312, \mathrm{P}=0.012)$ and lymph node metastasis (HR $=7.401,95 \%$ CI: 2.847-19.237, $\mathrm{P}<0.001$ ), but not total lesion size (for total lesion size $>10$ and $\leq 20 \mathrm{~mm}, \mathrm{P}=0.826$; for total lesion size $>20$ and $\leq 30 \mathrm{~mm}$, $\mathrm{P}=0.784)$ or CTR $(\mathrm{P}=0.753)$, were identified as independent predictive factors for decreased RFS (Table 4).

To study the impact of resection extent on prognosis, oncological outcomes after sublobar resections and lobectomy were first compared in 351 patients with PSNs of solid component size $\leq 20 \mathrm{~mm}$. No significant difference in 5 -year RFS was found after sublobar resections $(95.0 \%, 95 \%$ CI: 91.1-98.9\%) or after lobectomy (93.6\%, 95\% CI: 90.3$96.9 \%, \mathrm{P}=0.592$, Figure $4 A$ ). Among them, 112 patients had PSNs with solid component size $\leq 20 \mathrm{~mm}$ but total lesion size $>20$ and $\leq 30 \mathrm{~mm}$. Still, there was no significant difference in 5-year RFS after sublobar resections or lobectomy (88.9\%, 95\% CI: $77.1-100 \%$ vs. $90.0 \%$, $95 \%$ CI: 83.3-96.7\%, $\mathrm{P}=0.893$, Figure 4B).

\section{Discussion}

In this study, we found that compared to total lesion size, solid component size could better stratify prognosis and 
Table 2 Clinicopathological characteristics of part-solid nodules before propensity-score matching

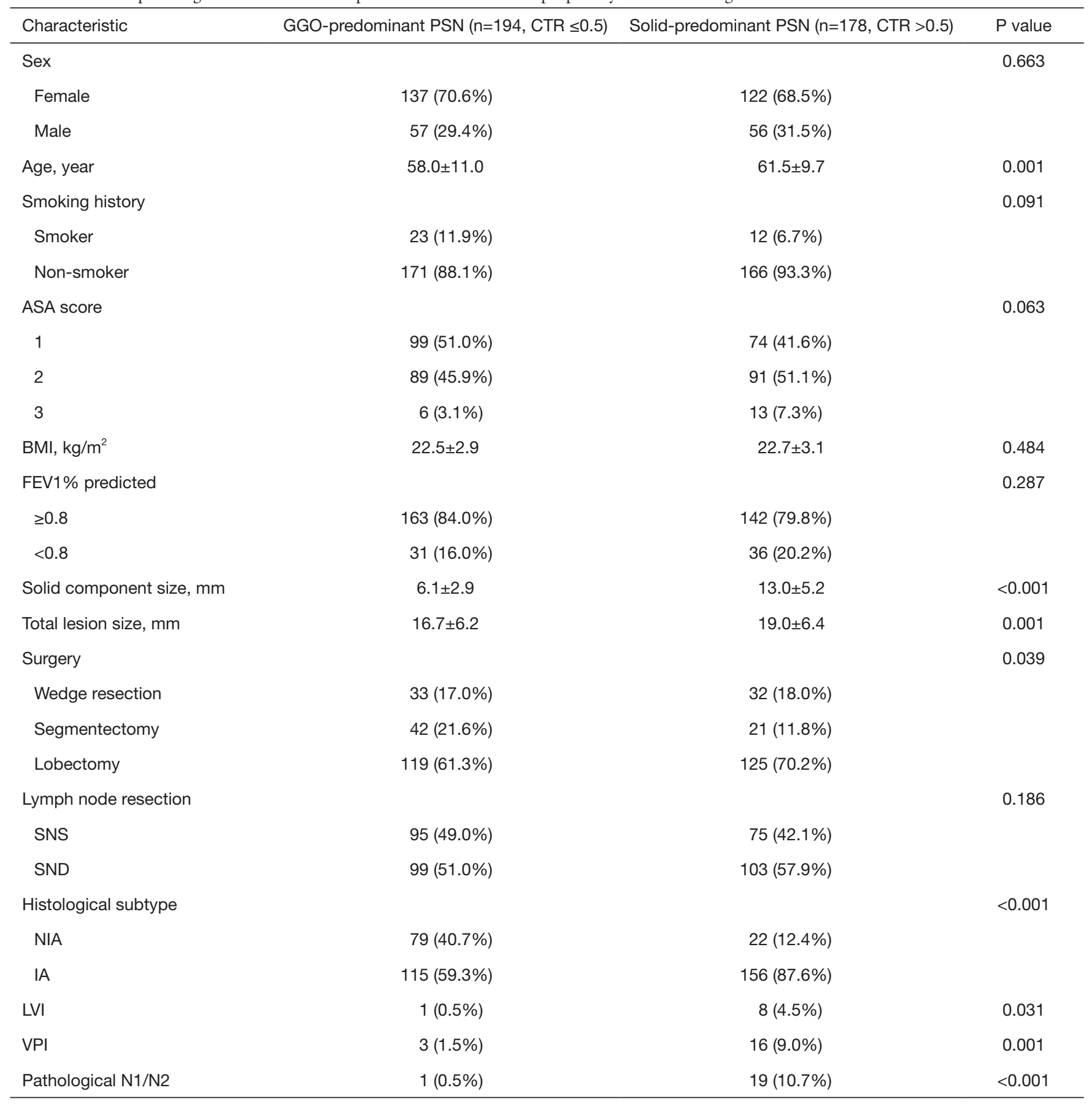

PSN, part-solid nodule; PSM, propensity-score matching; GGO, ground glass opacity; CTR, consolidation-to-tumor ratio; ASA, American Society of Anesthesiologists; BMI, body mass index; FEV1, forced expiratory volume in 1 second; SNS, systematic lymph node sampling; SND, systematic lymph node dissection; NIA, noninvasive adenocarcinoma; IA, invasive adenocarcinoma; LVI, lymphovascular invasion; VPI, visceral pleural invasion. 
Table 3 Clinicopathological characteristics of part-solid nodules after two propensity-score matching by total lesion size and solid component size, respectively

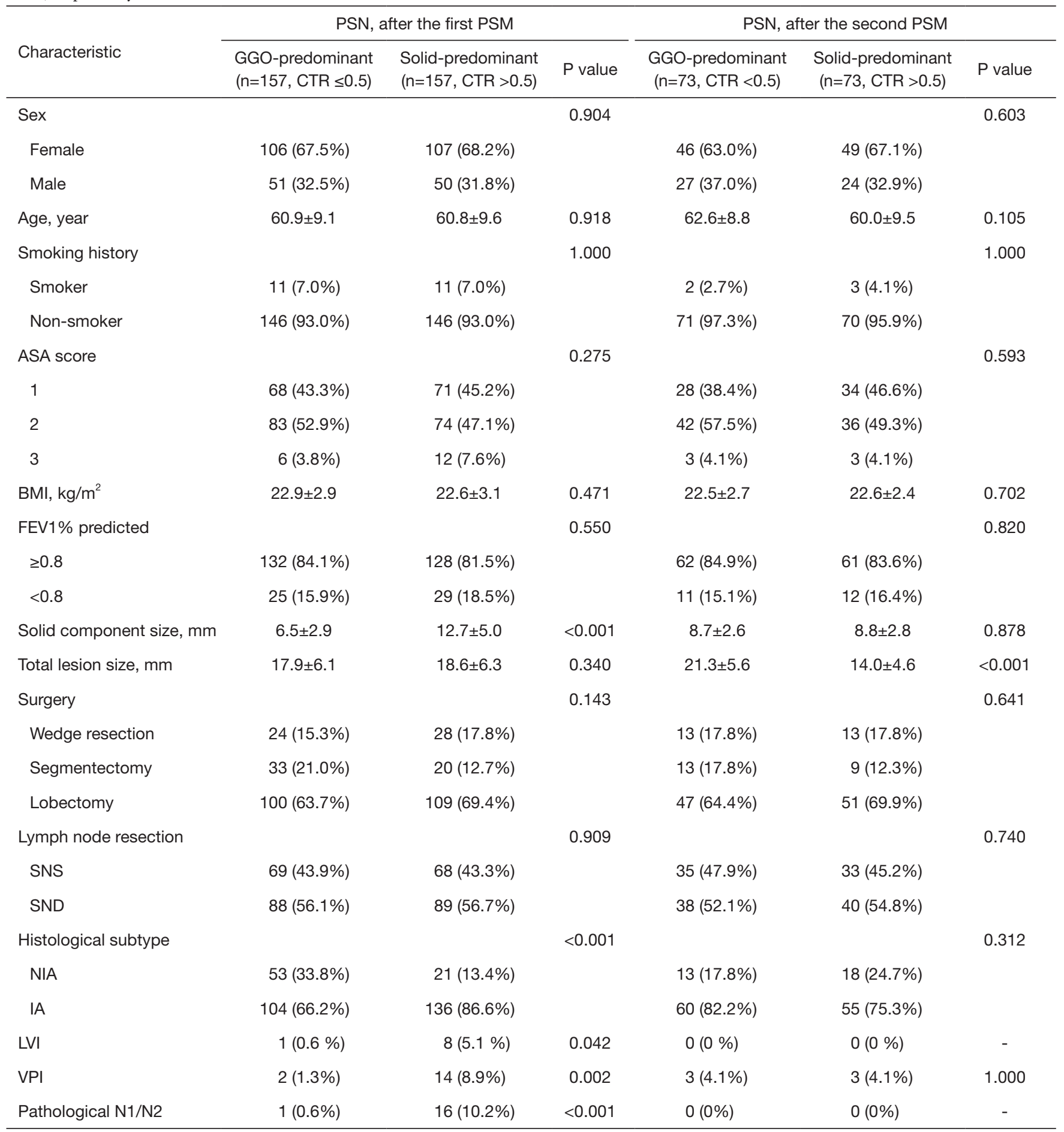

PSN, part-solid nodule; PSM, propensity-score matching; GGO, ground glass opacity; CTR, consolidation-to-tumor ratio; ASA, American Society of Anesthesiologists; BMI, body mass index; FEV1, forced expiratory volume in 1 second; SNS, systematic lymph node sampling; SND, systematic lymph node dissection; NIA, noninvasive adenocarcinoma; IA, invasive adenocarcinoma; LVI, lymphovascular invasion; VPI, visceral pleural invasion. 

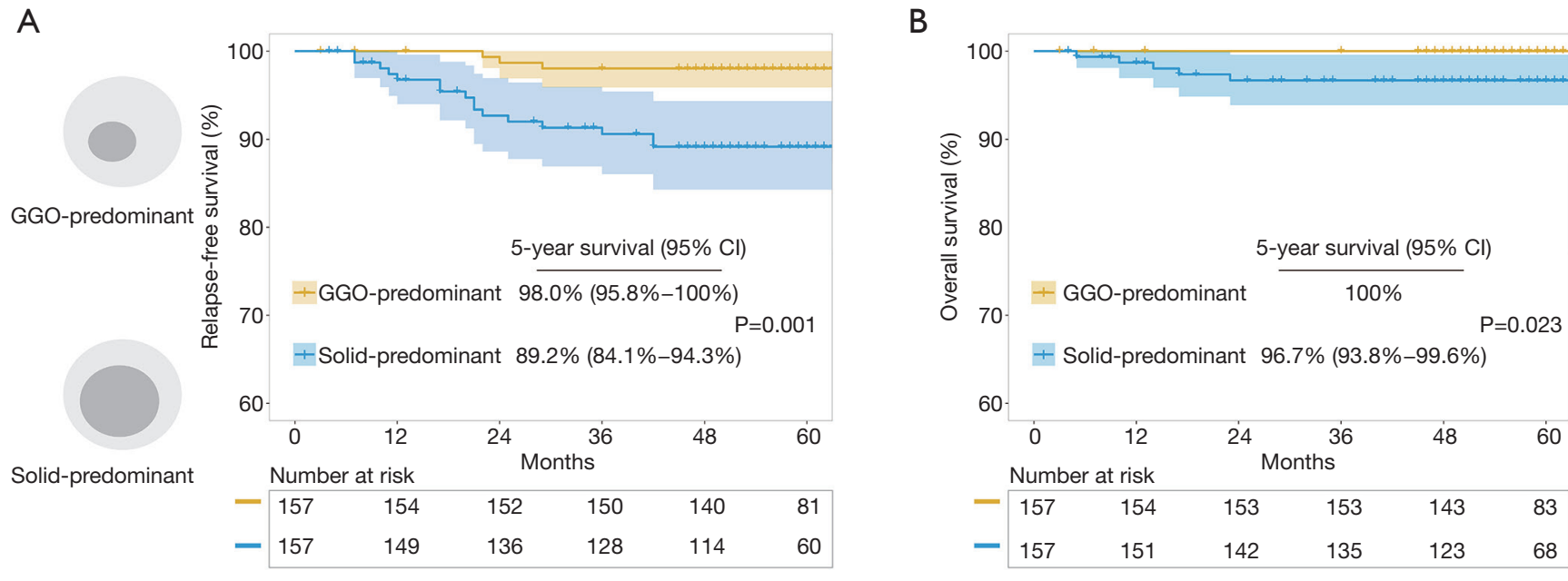

Figure 2 Comparison of survival between the GGO-predominant subgroup and the solid-predominant subgroup with similar total lesion size $\leq 30 \mathrm{~mm}$ after the first propensity-score matching. (A) For tumors with similar total lesion size, the solid-predominant subgroup had significantly worse 5-year relapse-free survival ( $\mathrm{P}=0.001)$ than the GGO-predominant subgroup; (B) for tumors with similar total lesion size, the solid-predominant subgroup had significantly worse 5 -year overall survival $(\mathrm{P}=0.023)$ than the GGO-predominant subgroup. GGO, ground glass opacity.

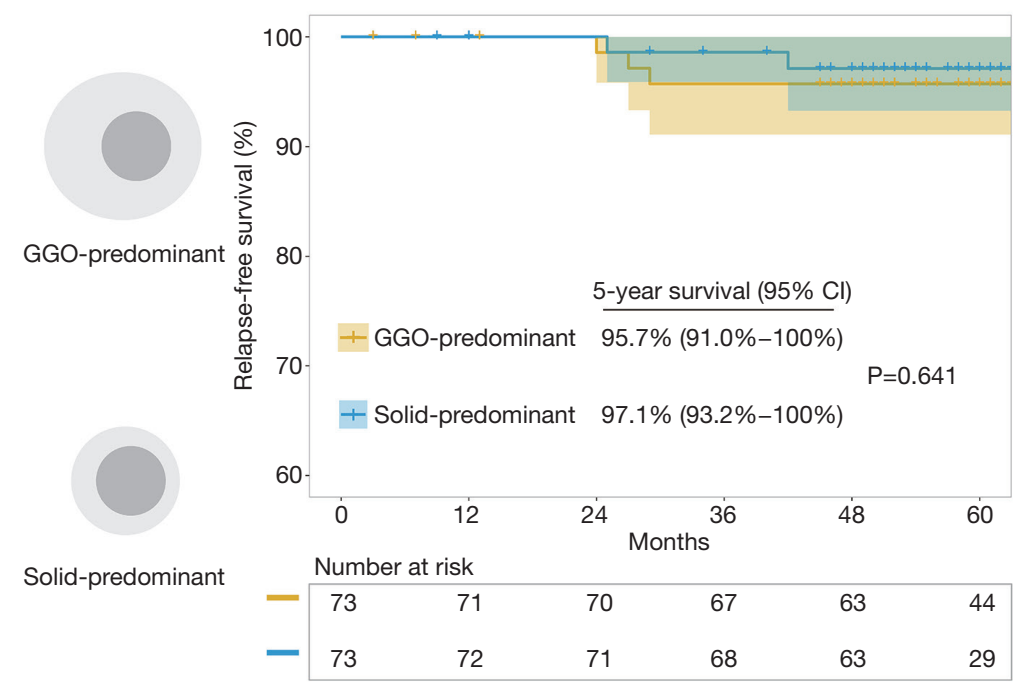

Figure 3 Comparison of relapse-free survival between the GGO-predominant subgroup and the solid-predominant subgroup with similar solid component size $\leq 20 \mathrm{~mm}$ after the second propensity-score matching. GGO, ground glass opacity.

histological characteristics for lung adenocarcinomas appearing as GGO-containing nodules on CT scan. Multivariate survival analysis also indicated that solid component size, but not total lesion size or CTR, was an independent prognostic factor for RFS. When using solid component size $\leq 20 \mathrm{~mm}$ as the selection criterion, sublobar resections did not show inferior efficacy to lobectomy in oncological outcomes, regardless of total lesion size. The results remained similar even for GGOcontaining tumors with solid component size $\leq 20 \mathrm{~mm}$ but total lesion size $>20 \mathrm{~mm}$.

Recent studies on resection extent for early-stage lung cancers have been focused on lesions with a total size less than $2 \mathrm{~cm}$. The LCSG trial (1) reported a $50 \%$ increase of 
Table 4 Univariate and multivariable Cox regression analysis of potential risk factors associated with relapse-free survival in part-solid nodules $(\mathrm{n}=372)$

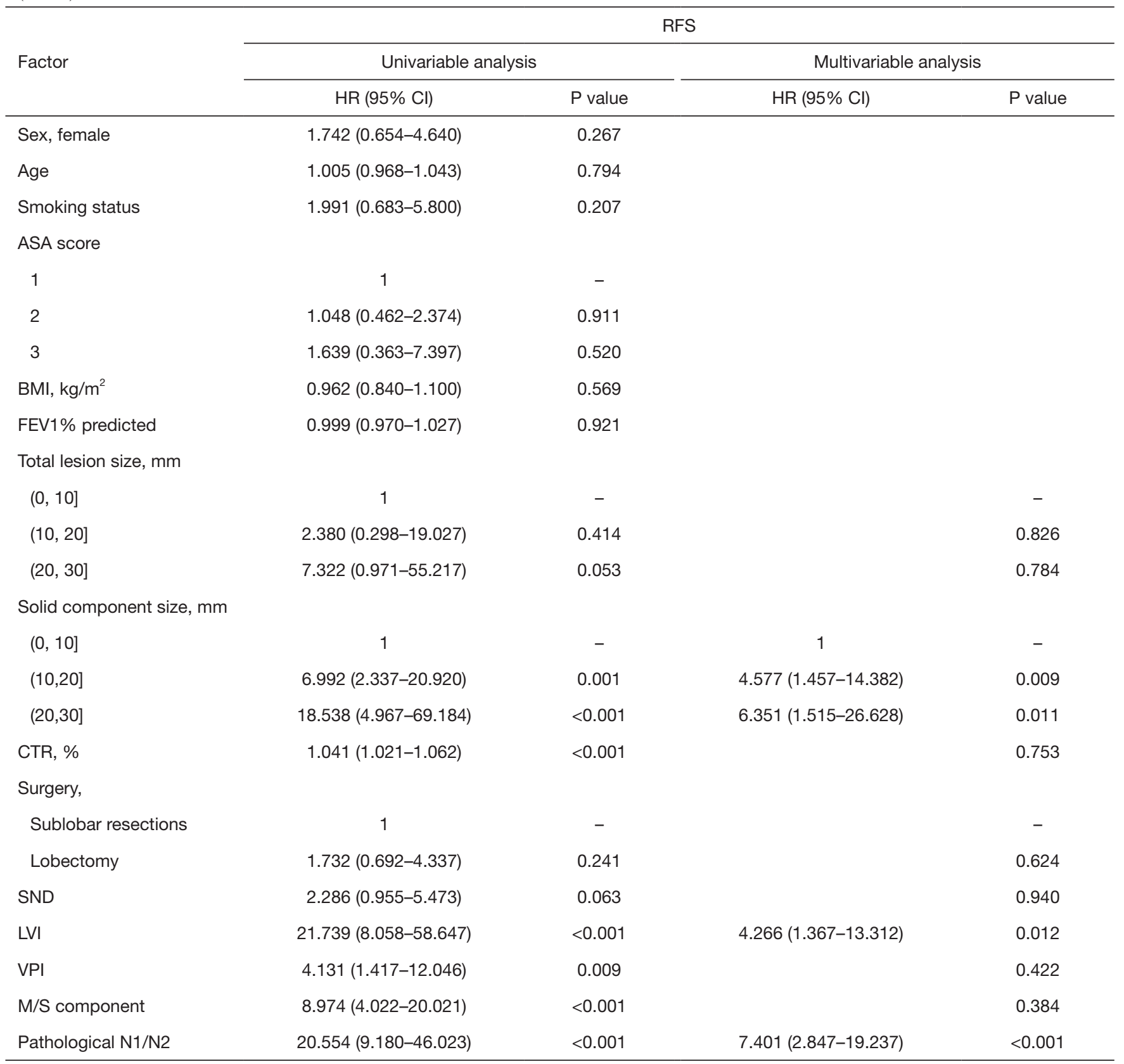

RFS, relapse-free survival; HR, hazard ratio; Cl, confidence interval; ASA, American Society of Anesthesiologists; BMI, body mass index; FEV1, forced expiratory volume in 1 second; CTR, consolidation-to-tumor ratio; SND, systematic lymph node dissection; LVI, lymphovascular invasion; VPI, visceral pleural invasion; M/S component, histological micropapillary or solid component.

cancer related death rate and a $75 \%$ increase of recurrence rate associated with sublobar resections. However, tumors $>2 \mathrm{~cm}$ were also included in that trial. With the widespread application of CT scan, tumors of smaller size were increasingly detected. And the current UICC/IASLC staging system has further broken down the T1 stage into 3 categories by $1 \mathrm{~cm}$ increment. Several retrospective studies have already observed similar oncological outcomes between lobectomy and sublobar resections in patients with small early-stage $\operatorname{NSCLC}(2,6,17,18)$. Zhao et al. 
A

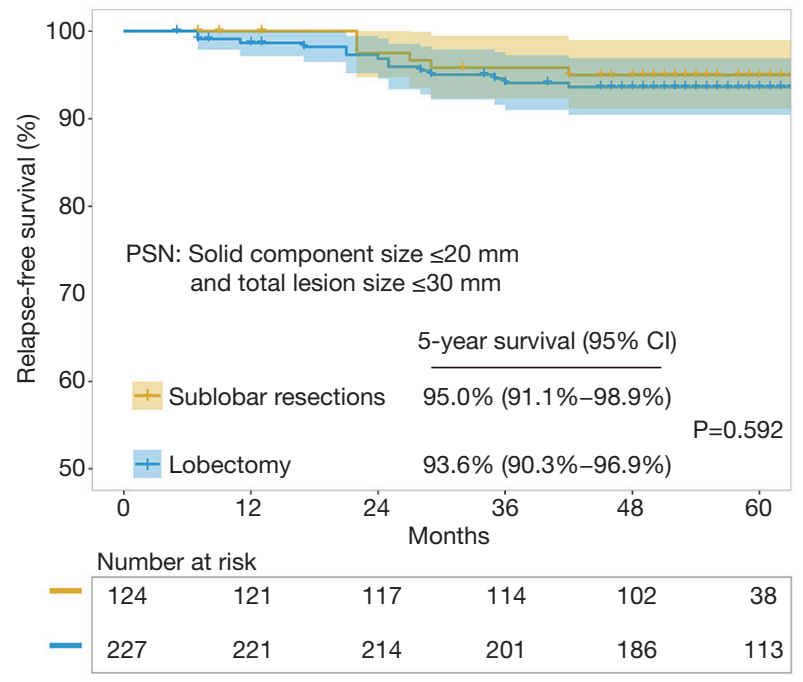

B

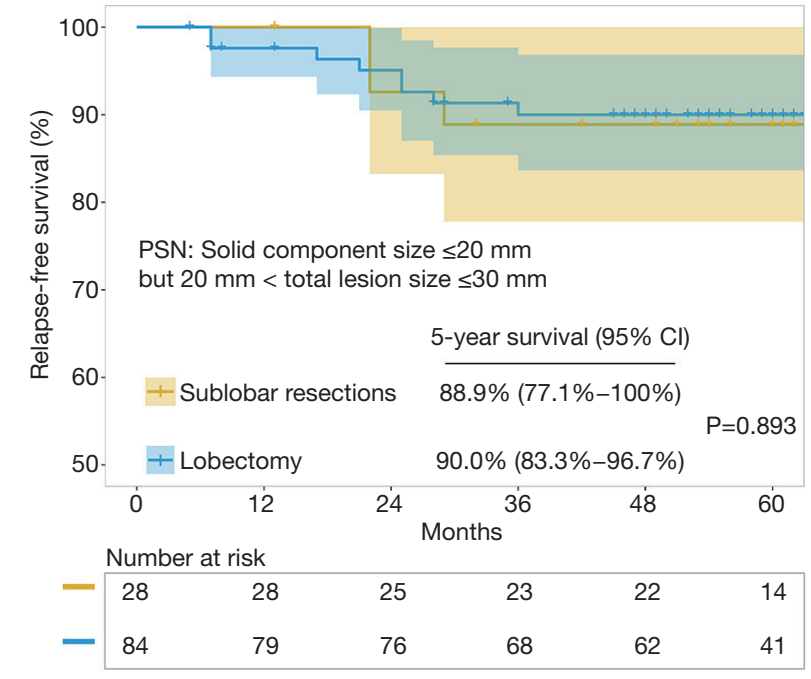

Figure 4 Relapse-free survival after sublobar resections or lobectomy in part-solid nodules (PSN) when solid component size was chosen as selection criterion for resection extent. (A) Sublobar resections showed comparable relapse-free survival to standard lobectomy ( $\mathrm{P}=0.592$ ) in PSNs with solid component size $\leq 20 \mathrm{~mm}$ and total lesion size $\leq 30 \mathrm{~mm}$; (B) a subgroup analysis: there was still no significant difference in relapse-free survival after sublobar resections or lobectomy $(\mathrm{P}=0.893)$ in part-solid nodules $(\mathrm{PSN})$ with solid component size $\leq 20$ mm but total lesion size $>20 \mathrm{~mm}$ and $\leq 30 \mathrm{~mm}$.

compared segmentectomy and lobectomy among patients with pathological stage IA adenocarcinomas $\leq 20 \mathrm{~mm}$ and demonstrated that segmentectomy was not associated with worse lung cancer-specific survival after PSM ( 5 -year survival $=83.89 \%$ for segmentectomy $v$ s. $86.11 \%$ for lobectomy) (18). Another prospective nonrandomized multicenter study reported that sublobar resection group had a similar oncological outcome with lobar resection group in peripheral cT1N0M0 NSCLC of $2 \mathrm{~cm}$ or less in size (2). The 5 -year DFS was $85.9 \%$ for sublobar resection group and $83.4 \%$ for lobar resection group ( $\mathrm{P}=0.2778)$. These results led to the change that sublobar resections may now be considered as an intentional treatment for tumors with a total lesion size of $20 \mathrm{~mm}$ or less in the clinical guideline for NSCLC (7). In the current study, we found that for tumors with similar solid component size $\leq 20 \mathrm{~mm}$ and similar resection extents, the GGO-predominant subgroup had similar histological characteristics and prognosis with the solid-predominant subgroup. Thus, a solid component size of $20 \mathrm{~mm}$ or less may be considered as a feasible criterion for guiding sublobar resections.

Another significant change brought by the use of CT screening was increased detection of GGO-containing nodules. It should be noted that not all patients included in the LCSG trial were diagnosed with adenocarcinomas. Neither was it known how many adenocarcinoma patients included had GGO-containing lesions representing a relatively indolent histology. Consequently, doubts were raised whether the results of the LCSG trial could also apply to these GGO-containing adenocarcinomas that have increasingly become the object of surgical treatment nowadays $(19,20)$. Recent studies have shown that patients with GGO-containing nodules especially those pure GGO lesions without solid component, had favorable prognosis after sublobar resections, even with a total lesion size $>20 \mathrm{~mm}$ (21-23). Our results were in accordance with these reports in that pure GGO had favorable histological characteristics and prognosis. We found that visceral pleural invasion and histological micropapillary or solid components were observed in merely $0.2 \%$ and $0.8 \%$ of patients in the pure GGO group, respectively. And there was no lymphovascular invasion or lymph node metastasis observed. Although $33.3 \%$ of patients with pure GGO $>20$ and $\leq 30 \mathrm{~mm}$ in size received sublobar resections, 5 -year OS was $100 \%$ in this group. These results indicated that sublobar resections were feasible for pure GGO lesions $\leq 30 \mathrm{~mm}$ when satisfactory parenchymal resection margin could be guaranteed. 
In terms of part-solid tumors, it was reported that radiological GGO and solid component usually correspond to lepidic pattern and invasive pattern, respectively, in histology (14). In the eighth edition of AJCC TNM staging system, $\mathrm{T}$ descriptor for part-solid tumors has been changed from total lesion size to solid component size (12). Previous studies also indicated that solid component size was a better prognostic factor than total lesion size. Su et al. (10) reported a better predictive value of solid component size for pathological malignant behaviors over total lesion size in clinical stage IA adenocarcinomas. Another propensity-score matched study (11) showed that although the total lesion size of the GGO-dominant lesions was larger than that of the solid-dominant lesions, no significant differences in prognosis were observed in node-negative adenocarcinomas with a similar invasive component size $<20 \mathrm{~mm}(\mathrm{P}=0.48$ for DFS and $\mathrm{P}=0.52$ for OS). We studied the prognosis and histological characteristics in a group of 372 patients with PSNs, using two PSM analyses. In PSNs with similar total lesion size but different solid component size after the first PSM, we found that the GGO-predominant subgroup had significantly better performance than the solid-predominant subgroup in both histological characteristics and prognosis. Then in PSNs with similar solid component size but different total lesion size after the second PSM, we found no significant difference in histological characteristics or prognosis between the GGO-predominant subgroup and the solid-predominant subgroup. These results indicated that the size of GGO had limited impact on the prognosis of GGO-containing tumors. And it was the solid component size rather than the total lesion size that predicted histological characteristics and prognosis in GGO-containing tumors. Consequently, it seems that total lesion size may not be an ideal selection criterion for sublobar resections in GGO-containing adenocarcinomas.

Comparison of sublobar resections and lobectomy is still underway, including two ongoing prospective randomized trials (CALGB 140503 and JCOG 0802). Although the study designs were somewhat different, total lesion size $\leq 2 \mathrm{~cm}$ was used as the inclusion criterion in both trials. The CALGB 140503 trial compares lobectomy with sublobar resections for tumors of total lesion size $\leq 2 \mathrm{~cm}$, without a definition on tumor histology or GGO component (9). The JCOG 0802 trial compares segmentectomy with lobectomy for tumors of total lesion size $\leq 2 \mathrm{~cm}$ and CTR $>0.5$ (8). But the concept of CTR could not be translated into the current TNM staging of lung cancers, as tumors with similar solid component size might be grouped to different CTR categories. Our results showed that in accordance with the current TNM staging, solid component size performed better than total lesion size on predicting histological characteristics and prognosis. In the current study, when using solid component size as the selection criterion, we found a similar 5-year RFS at 95.0\% (95\% CI: 91.1-98.9\%) after sublobar resections and 93.6\% (95\% CI: 90.3-96.9\%, $\mathrm{P}=0.592)$ after lobectomy in PSNs with solid component size $\leq 20 \mathrm{~mm}$. These include patients having $\mathrm{T} 1 \mathrm{a}$ or $\mathrm{T} 1 \mathrm{~b}$ tumors with total lesion size $\leq 20 \mathrm{~mm}$ that would meet the inclusion criteria in both the JCOG 0802 trial and the CALGB 140503 trial. Then for tumors with a total lesion size $>20 \mathrm{~mm}$ that would not have been included in either of those two trials or are not indicated for sublobar resections according to the current guidelines of total lesion size $\leq 20 \mathrm{~mm}$, it remains to be investigated whether sublobar resections are still non-inferior to lobectomy if the lesion is GGO-containing and with a solid component size less than $20 \mathrm{~mm}$. We thus compared the oncological outcomes in PSNs containing solid component $\leq 20 \mathrm{~mm}$ but with a total lesion size $>20$ and $\leq 30 \mathrm{~mm}$. We found that sublobar resections also showed comparable oncological outcomes to standard lobectomy. Our results were in accordance with what was reported by Hattori et al. (24) that sublobar resections might still be feasible as long as the tumor is GGO containing. Consequently, these results indicate that solid component size is a more appropriate selection criterion for sublobar resections in GGO-containing lung adenocarcinomas.

We acknowledged that this study had several limitations. First, the data was retrieved retrospectively from a prospectively kept database of a single institution, which inevitably had intrinsic biases. Second, although PSM was helpful in eliminating potential confounding factors, the sample size was reduced to some extent. Third, there were significant differences in histological characteristics and prognosis between the 157 matching pairs of GGOpredominant and solid-predominant nodules of similar total lesion size, but not between the 73 matching pairs of nodules with similar solid component sizes. This should be interpreted with caution because of a more robust statistical power in the former analysis. Moreover, resection margin is considered as an important factor relating to prognosis after sublobar resections for NSCLC. But we were unable to examine the relationship between resection margin and the outcomes because of the retrospective nature of our study. However, our study provides useful information on how future prospective trials should be designed in order to 
better define the appropriate resection extent for early stage GGO-containing lung adenocarcinomas.

In conclusion, our results showed that solid component size better predicts histological characteristics and prognosis than total lesion size in early-stage GGO-containing lung adenocarcinomas, and ensures similar outcomes after lobectomy or sublobar resections in such patients. Thus, solid component size instead of total lesion size may be a more appropriate selection criterion for sublobar resections. Future studies on resection extent for early-stage lung cancers should be based on solid component size rather than total lesion size so that their results could better direct clinical practice.

\section{Acknowledgments}

The authors thank Yuan Liu, from the Statistics Center, Shanghai Chest Hospital, Shanghai Jiao Tong University for helping with statistical analysis of the data in this study. And we also thank Fenghao Yu and Xiuxiu Hao from Department of Thoracic Surgery, Shanghai Chest Hospital, Shanghai Jiao Tong University for their help with language polishing.

Funding: None.

\section{Footnote}

Reporting Checklist: The authors have completed the STROBE reporting checklist. Available at http://dx.doi. org/10.21037/tlcr-21-132

Data Sharing Statement: Available at http://dx.doi. org/10.21037/tlcr-21-132

Conflicts of Interest: All authors have completed the ICMJE uniform disclosure form (available at http://dx.doi. org/10.21037/tlcr-21-132). The authors have no conflicts of interest to declare.

Ethical Statement: The authors are accountable for all aspects of the work in ensuring that questions related to the accuracy or integrity of any part of the work are appropriately investigated and resolved. The study was conducted in accordance with the Declaration of Helsinki (as revised in 2013). This study was approved by the institutional review board of the Shanghai Chest Hospital [No. KS(Y)1668] and informed consent was waived due to the retrospective nature of the study.
Open Access Statement: This is an Open Access article distributed in accordance with the Creative Commons Attribution-NonCommercial-NoDerivs 4.0 International License (CC BY-NC-ND 4.0), which permits the noncommercial replication and distribution of the article with the strict proviso that no changes or edits are made and the original work is properly cited (including links to both the formal publication through the relevant DOI and the license). See: https://creativecommons.org/licenses/by-nc-nd/4.0/.

\section{References}

1. Ginsberg RJ, Rubinstein LV. Randomized trial of lobectomy versus limited resection for T1 N0 non-small cell lung cancer. Lung Cancer Study Group. Ann Thorac Surg 1995;60:615-22; discussion 622-3.

2. Okada M, Koike T, Higashiyama $M$, et al. Radical sublobar resection for small-sized non-small cell lung cancer: a multicenter study. J Thorac Cardiovasc Surg 2006;132:769-75.

3. Kodama K, Doi O, Higashiyama $M$, et al. Intentional limited resection for selected patients with T1 N0 M0 non-small-cell lung cancer: a single-institution study. J Thorac Cardiovasc Surg 1997;114:347-53.

4. Chiang XH, Hsu HH, Hsieh MS, et al. PropensityMatched Analysis Comparing Survival After Sublobar Resection and Lobectomy for cT1N0 Lung Adenocarcinoma. Ann Surg Oncol 2020;27:703-15.

5. Keenan RJ, Landreneau RJ, Maley RH Jr, et al. Segmental resection spares pulmonary function in patients with stage I lung cancer. Ann Thorac Surg 2004;78:228-33; discussion 233.

6. Landreneau RJ, Normolle DP, Christie NA, et al. Recurrence and survival outcomes after anatomic segmentectomy versus lobectomy for clinical stage I nonsmall-cell lung cancer: a propensity-matched analysis. J Clin Oncol 2014;32:2449-55.

7. National Comprehensive Cancer Network (NCCN) clinical practice guidelines in Oncology: Non-Small Cell Lung Cancer (2021.Version 2). Available online: https:// www.nccn.org

8. Nakamura K, Saji H, Nakajima R, et al. A phase III randomized trial of lobectomy versus limited resection for small-sized peripheral non-small cell lung cancer (JCOG0802/WJOG4607L). Jpn J Clin Oncol 2010;40:271-4.

9. Altorki NK, Wang X, Wigle D, et al. Perioperative mortality and morbidity after sublobar versus lobar 
resection for early-stage non-small-cell lung cancer: post-hoc analysis of an international, randomised, phase 3 trial (CALGB/Alliance 140503). Lancet Respir Med 2018;6:915-24.

10. Su H, Dai C, She Y, et al. Which T descriptor is more predictive of recurrence after sublobar resection: whole tumour size versus solid component size? Eur J Cardiothorac Surg 2018;54:1028-36.

11. Han SJ, Jeon JH, Jung W, et al. Do ground-glass opacitydominant features have prognostic significance in nodenegative adenocarcinomas with invasive components of similar sizes? Eur J Cardiothorac Surg 2020;57:1189-94.

12. Travis WD, Asamura H, Bankier AA, et al. The IASLC Lung Cancer Staging Project: Proposals for Coding T Categories for Subsolid Nodules and Assessment of Tumor Size in Part-Solid Tumors in the Forthcoming Eighth Edition of the TNM Classification of Lung Cancer. J Thorac Oncol 2016;11:1204-23.

13. Hansell DM, Bankier AA, MacMahon H, et al. Fleischner Society: glossary of terms for thoracic imaging. Radiology 2008;246:697-722.

14. Travis WD, Brambilla E, Noguchi M, et al. International association for the study of lung cancer/american thoracic society/european respiratory society international multidisciplinary classification of lung adenocarcinoma. J Thorac Oncol 2011;6:244-85.

15. Lardinois D, De Leyn P, Van Schil P, et al. ESTS guidelines for intraoperative lymph node staging in non-small cell lung cancer. Eur J Cardiothorac Surg 2006;30:787-92.

16. Zhao W, Chen T, Feng J, et al. Comparison of lymph node dissection and lymph node sampling for non-small

Cite this article as: Lin B, Wang R, Chen L, Gu Z, Ji C, Fang W. Should resection extent be decided by total lesion size or solid component size in ground glass opacity-containing lung adenocarcinomas? Transl Lung Cancer Res 2021;10(6):24872499. doi: $10.21037 /$ tlcr-21-132 cell lung cancers by video-assisted thoracoscopic surgery. J Thorac Dis 2019;11:505-13.

17. Tsutani Y, Miyata Y, Nakayama H, et al. Oncologic outcomes of segmentectomy compared with lobectomy for clinical stage IA lung adenocarcinoma: propensity score-matched analysis in a multicenter study. J Thorac Cardiovasc Surg 2013;146:358-64.

18. Zhao ZR, Situ DR, Lau RWH, et al. Comparison of Segmentectomy and Lobectomy in Stage IA Adenocarcinomas. J Thorac Oncol 2017;12:890-6.

19. Sakurai H, Asamura H. Sublobar resection for early-stage lung cancer. Transl Lung Cancer Res 2014;3:164-72.

20. Nakazawa S, Shimizu K, Mogi A, et al. VATS segmentectomy: past, present, and future. Gen Thorac Cardiovasc Surg 2018;66:81-90.

21. Mao R, She Y, Zhu E, et al. A Proposal for Restaging of Invasive Lung Adenocarcinoma Manifesting as Pure Ground Glass Opacity. Ann Thorac Surg 2019;107:1523-31.

22. Lim HJ, Ahn S, Lee KS, et al. Persistent pure ground-glass opacity lung nodules $>/=10 \mathrm{~mm}$ in diameter at CT scan: histopathologic comparisons and prognostic implications. Chest 2013;144:1291-9.

23. Moon Y, Lee KY, Park JK. The prognosis of invasive adenocarcinoma presenting as ground-glass opacity on chest computed tomography after sublobar resection. J Thorac Dis 2017;9:3782-92.

24. Hattori A, Matsunaga T, Takamochi K, et al. The oncological outcomes of segmentectomy in clinical-T1b lung adenocarcinoma with a solid-dominant appearance on thin-section computed tomography. Surg Today 2016;46:914-21. 